\title{
Defensins defend against bone loss
}

\author{
Friedrich C. Luft $^{1}$
}

Published online: 6 October 2017

(C) Springer-Verlag GmbH Germany 2017

Defensins are small, cysteine-rich cationic proteins contained within cells of the immune system. The defensin peptides assist in killing phagocytosed bacteria in neutrophils and in many epithelial cell types. Most defensins function by binding to the microbial cell membrane, and, once embedded, forming pore-like membrane defects that allow efflux of essential ions and nutrients. The term "defensin" was coined in the 1980s, although perhaps the term "cationic antimicrobial proteins" would be better [1]. The underlying genes responsible for defensin production are highly polymorphic. Some aspects are conserved; however, the hallmarks of defensins are their small size, high density of cationic charge, and a six-cysteineresidue motif. In humans, two subfamilies of defensins are important, namely a-defensin and $\beta$-defensin. The $\beta$ defensins are induced by different stimuli, including lipopolysaccharide, lipoteichoic acid, and bacterial DNA [2].

It appears that defensins can defend against more than just outside invaders. For instance, a defensin-like peptide is the cathelicidin-related antimicrobial peptide (CRAMP). CRAMP is expressed in salivary glands and in the oral mucosa. In Kostmann's disease, a severe form of congenital neutropenia, CRAMP is deficient. The patients develop severe infections. However, they also feature remarkable loss of alveolar bone within the oral cavity. This connection suggested that CRAMP could possibly prevent the progression of periodontal disease, a hypothesis that has been addressed earlier.

Friedrich C. Luft

luft@ charite.de

Experimental and Clinical Research Center, a joint cooperation between the Charité Medical Faculty and the Max Delbrück Center for Molecular Medicine, Lindenberger Weg 80,

13125 Berlin, Germany
Although the earlier studies were not entirely convincing, the idea was proposed that CRAMP and the human analog LL-37 could serve as an osteoblast-derived protector in bacterial infection-induced osteoclastic bone reabsorption $[3,4]$. In this issue of $J \mathrm{Mol} \mathrm{Med}$, Park and colleagues pursue this idea further by investigating human $\beta$-defensin-3 C-15 (HBD3) and any role that small peptide could have in inhibiting bone reabsorption by impairing osteoclast activity [5].

The authors first examined the effect of HBD3 on receptor activator of nuclear factor $\mathrm{K}-\mathrm{B}$ ligand (RANKL). RANKL has been identified to affect the immune system and control bone regeneration and remodeling. RANKL is an apoptosis regulator, a binding partner of osteoprotegerin (OPG), and a ligand for the receptor activator of nuclear factor $K-B$ (RANK). RANKL binds to RANK, which then binds to tumor necrosis factor (TNF)receptor associated factor-6 (TRAF6). TRAF6 stimulates the activation of the c-jun N-terminal kinase (JNK) and nuclear factor к-B (NF- $\mathrm{B}$ ) pathways, which trigger differentiation and activation of multinucleated osteoclasts. The investigators found that RANKL increased the number of multinucleated osteoclast-like cells as expected. However, HBD3 inhibited the RANKLincreased TRAP-positive multinucleated cell formation (Fig. 1). In addition, HBD3 disrupted the formation of the RANKLinduced podosome belt, which is a feature typically found in mature osteoclasts with bone-resorbing capacity.

Once activated, osteoclasts move to areas of microfracture in bone by chemotaxis. Lacunae are small spaces within bone containing an osteocyte in bone or chondrocyte in cartilage. They were named by John Howship (1781-1841), a British surgeon who first identified the structures. Osteoclasts can also reside in Howship's lacunae, formed from the digestion of the underlying bone. The sealing zone is the attachment of the osteoclast's plasma membrane to the underlying bone. Sealing zones are bounded by belts of specialized adhesion 


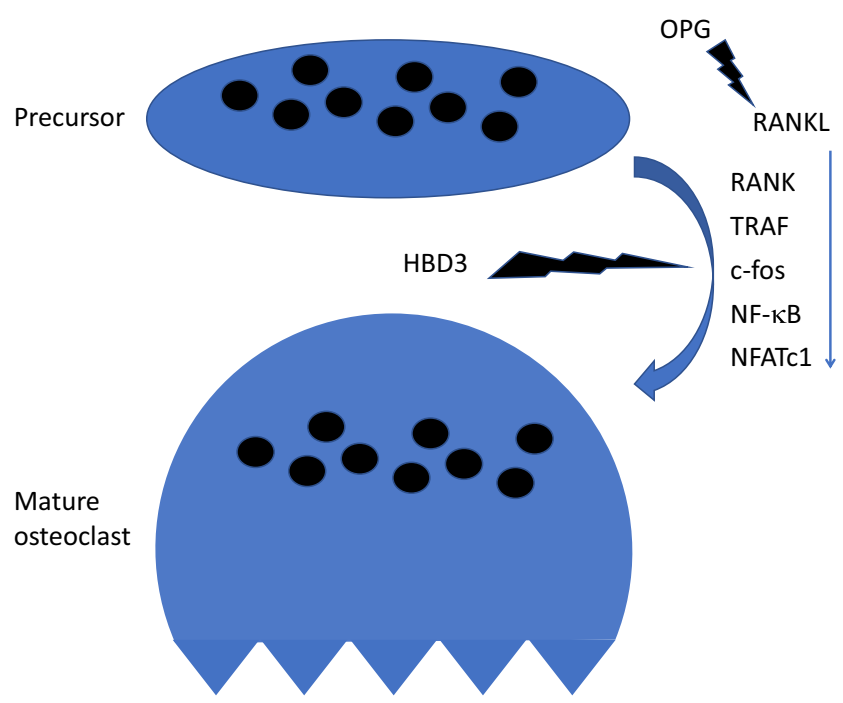

Fig. 1 Osteoclast formation requires the presence of receptor activator of nuclear factor- $\mathrm{kB}$ ligand (RANKL) and macrophage colony-stimulating factor (M-CSF). These membrane-bound proteins are produced by neighboring stromal cells and osteoblasts, thus requiring direct contact between these cells and osteoclast precursors. RANKL binds to tumor necrosis factor (TNF)-receptor associated factor-6 (TRAF6). TRAF activates NF-KB and NFATc1 and c-Fos through receptor activator of nuclear factor- $\mathrm{KB}$ (RANK). Osteoclast differentiation is inhibited by osteoprotegerin (OPG), which is produced by osteoblasts and binds to RANKL thereby preventing interaction with RANK. While osteoclasts are derived from the hematopoietic lineage, osteoblasts are derived from mesenchymal stem cells. Human $\beta$-defensin-3 (HBD3) that can be produced by osteoblasts inhibits this progression

structures called podosomes. HBD3 apparently downregulated a series of signaling molecules that are involved in the podosome-belt formation. More importantly, HBD3 reduced bone loss in a mouse calvarium-implantation model. When the authors used a bacterial model involving Aggregatibacter actinomycetemcomitans-induced lipopolysaccharide, the administration of HBD3 inhibited the effect. The authors suggest that HBD3 acts as an anti-reabsorption agent in bone, and perhaps, the principle could be used in the treatment of osteoporosis or periodontal disease. The data are consistent with a downregulation of nuclear factor of activated T cells (NFATc1) and cFos, transcription-factor mechanisms that are stimulators of osteoclast differentiation.

Interestingly, HBD 3 can be produced in healthy bone by osteoblasts. In an earlier study, samples of healthy and osteomyelitic human bone were assessed for the expression of HBD3. In that study, bacteria rapidly and effectively induced osteoblastic HBD3 production [6]. However, much remains to be learned about the mechanisms involved and whether or not HBD3 requires the presence of a bacterial presence for its effects. As an anti-osteoporosis treatment,
HBD3 would have to join a series of active competitors. Bisphosphonates block osteoclast activity; selective estrogen receptor modulators (SERMs) also inhibit bone reabsorption. Furthermore, existing and upcoming treatments affect remodeling as well as modeling [7]. Teriparatide, a recombinantDNA form of parathyroid hormone consisting of the first 34 amino acids, stimulates bone formation, $70 \%$ of which is remodeling based and $20-30 \%$ is modeling based. The vast majority of modeling represents overflow from remodeling units rather than de novo modeling. Denosumab, a human monoclonal antibody that blocks RANKL, inhibits bone remodeling but is permissive for modeling at cortex. Odanacatib inhibits bone resorption by inhibiting cathepsin $\mathrm{K}$ activity, whereas modeling-based bone formation is stimulated at periosteal surfaces. Inhibition of sclerostin stimulates bone formation and histomorphometric analysis demonstrated that bone formation is predominantly modeling based. Sclerostin, a bone morphogenic protein family member, is produced by the osteocyte and has anti-anabolic effects on bone formation. A single dose of anti-sclerostin antibody, romosozumab, increased bone density in the hip and spine in healthy men and postmenopausal women, and the drug was well tolerated. However, further additions to the therapeutic armamentarium, including HBD3, would be welcome.

Respectfully,

Friedrich C. Luft

\section{References}

1. Lehrer RI (2004) Primate defensins. Nat Rev Microbiol 2:727-738

2. Selsted ME, Ouellette AJ (2005) Mammalian defensins in the antimicrobial immune response. Nat Immunol 6:551-557

3. Supanchart C, Thawanaphong S, Makeudom A, Bolscher JG, Nazmi K, Kornak U, Krisanaprakornkit S (2012) The antimicrobial peptide, LL-37, inhibits in vitro osteoclastogenesis. J Dent Res 91:1071-1077

4. Horibe K, Nakamichi Y, Uehara S, Nakamura M, Koide M, Kobayashi Y, Takahashi N, Udagawa N (2013) Roles of cathelicidin-related antimicrobial peptide in murine osteoclastogenesis. Immunology 140:344-351

5. Park OJ, Kim J, Ahn KB, Lee JY, Park YJ, Kum KY, Yun CH, Han SH (2017) A 15-amino acid C-terminal peptide of beta-defensin-3 inhibits bone resorption by inhibiting the osteoclast differentiation and disrupting podosome belt formation. J Mol Med (Berl) https:// doi.org/10.1007/s00109-017-1589-2133

6. Varoga D, Wruck CJ, Tohidnezhad M, Brandenburg L, Paulsen F, Mentlein R, Seekamp A, Besch L, Pufe T (2009) Osteoblasts participate in the innate immunity of the bone by producing human beta defensin-3. Histochem Cell Biol 131:207-218

7. Langdahl B, Ferrari S, Dempster DW (2016) Bone modeling and remodeling: potential as therapeutic targets for the treatment of osteoporosis. Ther Adv Musculoskelet Dis 8:225-235 